\section{EARLY-WARNING SYSTEMS}

\section{Detecting famine from space}

Earths Future http://doi.org/dpxr (2020)

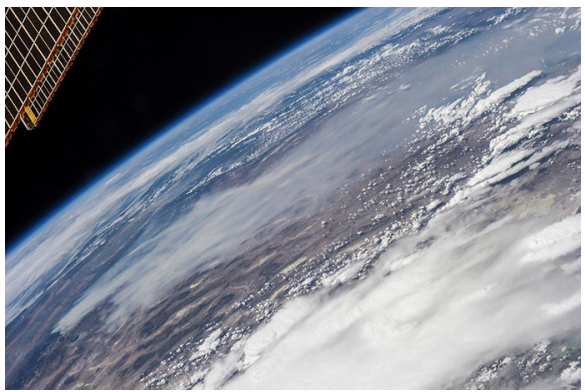

Credit: Stocktrek Images / Stocktrek Images / Getty

The frequency and severity of droughts is expected to increase under climate change with implications not only for terrestrial vegetation and carbon storage but also for human societies. In particular, drought can increase the risk for famine in places already vulnerable, for example, due to weaknesses in food distribution systems. At present, most early-warning systems for famine are based on local information, such as crop assessments and household data, which can be expensive and time consuming to process.

Krishna Krishnamurthy from the University of California Los Angeles, USA, and colleagues explore the potential to use remote-sensing data as an early-warning system using tipping point theory to detect the shift to a state of food crisis. They identify satellite datasets with a global scale and at fine enough spatial and temporal resolution to be useful for famine response. Although there are limitations, data on snow, soil moisture, precipitation, groundwater, evapotranspiration, vegetation health and chlorophyll fluorescence are especially promising. Such an approach could improve existing warning systems under climate change.

https://doi.org/10.1038/s41558-020-0748-6

\section{HIBERNATION BEHAVIOUR}

\section{Bats seek cold roosts}

PLoS ONE 15, e0227912 (2020)

For hibernating mammals, increases in yearly temperature can impact hibernation quality, leading to subsequent fitness fallouts. For bats, hibernating at warmer temperatures requires increased energy expenditure, which can result in lowered survival or reproductive success. While previous studies have indicated that warming may promote range changes for some bat species, the impact on cryophilic (cold loving), non-migrating species which may be particularly susceptible to warming - is not well understood.

Iwona Gottfried at the University of Wroclaw, Poland, and colleagues monitored hibernating cryophile Barbastella barbastellus populations in the 15 largest hibernacula and in 47 small bunkers across Poland. While the total population remained relatively stable across the 13-year study period, a significant negative correlation was observed between the number of individuals hibernating in hibernacula and mean winter temperature. By contrast, warmth correlated with an increase in individuals inhabiting the less-climatically buffered bunkers, suggesting that bats make behavioural changes to keep cool. These results have

\section{CLIMATE POLICY}

\section{Stranded investments}

J. Environ. Econ. Manag. 100, 102277 (2020).

Financial investments in fossil fuel assets could be lost if climate policies limit emissions in line with climate targets. Stranded assets, as these are known, thus represent an investment risk which may not be currently accounted for by investors and company valuations.

The German climate policy Klimabeitrag - the climate levy to reduce coal-generated electricity - allowed Suphi Sen and Marie-Theres von Schickfus of University of Munich, to investigate how investors consider and price such risk of stranding on German utility companies. The policy had three stages - introduction of the levy on carbon emissions, inclusion of a compensation mechanism and regulatory compliancy checks of the compensation mechanism - and the authors tracked stock market response to each stage.

The first two stages did not result in significant reactions, whereas the third stage elicited a significant negative reaction. This pattern of reaction is suggestive that investors had priced in compensation rather than overlooked the risk of asset stranding. Thus, steep and rapid devaluation of fossil fuel companies could result from climate policy changes where compensation is not included.

https://doi.org/10.1038/s41558-020-0751-y implications for bat hibernation in a warming Poland and affect current methods for monitoring bat numbers.

https://doi.org/10.1038/s41558-020-0750-z

\section{ATMOSPHERIC CHEMISTRY} III-sooted models Proc. Natl Acad. Sci. USA 117, 5196-5203 (2020)

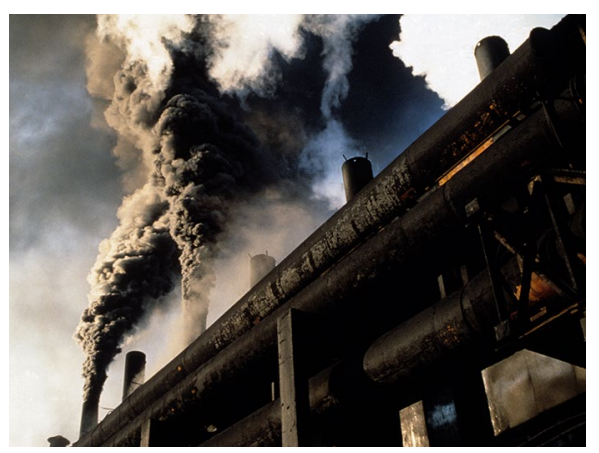

Credit: Andrew Holbrooke / Contributor / Corbis Historical / Getty

Atmospheric black carbon (BC) or soot - formed by the incomplete combustion of fossil fuels, biofuel and biomass causes warming by absorbing sunlight and enhancing the direct radiative forcing of the climate. As BC ages, it is coated with material due to gas condensation and collisions with other particles. These processes lead to variation in the composition of BC-containing particles and in the arrangement of their internal components a mixture of $\mathrm{BC}$ and other material - though global climate models do not fully account for these heterogeneities. Instead, BC-containing particles are typically modelled as uniformly coated spheres with identical aerosol composition, and these simplifications lead to overestimated absorption.

Laura Fierce of Brookhaven National Laboratory, USA, and colleagues exploit laboratory and field measurements to empirically update this common model approximation. By accounting for how material is arranged within BC-containing particles and, more importantly, the variability in their internal composition, the authors resolve these persistent model discrepancies. These results offer a framework for improving climate models and providing better estimates of the historical and future climate impacts of BC. 